\title{
Epidemiological Model Suggests D614G Spike Protein Mutation Accelerates Transmission of COVID-19 - Worldwide, 2020
}

\author{
Liang Wang'; Yuhai Bi ${ }^{1,2, *} ;$ George F. Gao ${ }^{1,2, *}$
}

The coronavirus disease 2019 (COVID-19) pandemic was caused by a novel type of coronavirus also known as SARS-CoV-2, 2019-nCoV, and HCoV-19 (1) and quickly spread around the world becoming a major threat to public health (2-3). Although COVID-19 virus is characterized as possessing a large genome and having limited genetic diversity (4) like other coronaviruses due to the high fidelity of its replication mechanism, many single nucleotide polymorphisms (SNPs) have been detected so far. Among the reported SNPs, the strain with the G614 mutation in the $S$ protein has replaced the strain containing D614 as the world's primary pandemic strain (5). However, it was still unclear if COVID-19 virus strains with G614 were more transmissible than those with D614 (6).

We reconstructed several transmission chains of COVID-19 during the early epidemic phase for 3 countries (Australia, the UK, and USA) based on genomic data from GISAID ( 7 ) and Bayesian inference under an epidemiological model for strains with D614 and G614 due to the similar amount of genomes within each country (Figure 1A). Then we inferred the $R_{0}$ in those transmission chains to compare the difference of transmissibility among humans between D614 and G614 (see the Supplementary Material for details, available in http://weekly.chinacdc.cn/). The $R_{0}$ caused by G614 was significantly higher than that caused by D614 in Australia and USA, as there was no intersection of $95 \%$ confidence intervals (CI) of the 2 distributions of $R_{0}$ (Figure 1B). For the UK, the mean value of $R_{0}$ for G614 was slightly higher than D614. For G614, its mean value of $R_{0}$ was outside the $95 \%$ CI of the estimated $R_{0}$ of D614. The mean value of $R_{0}$ of D614 also was outside the $95 \%$ CI of the estimated $R_{0}$ of G614. In addition, the $R_{0}$ for D614 was similar among three countries (from 1.56 for USA to 1.73 for Australia). However, significantly different $R_{0}$ was detected among countries (from 1.82 for the UK to 3.87 for USA), indicating that the spatial transmission of G614 had higher variation than D614.

Since the data used in this study were all collected during the early phases of each countries' outbreak and no stringent prevention and control strategies were implemented in these three countries in those phases, the estimation of $R_{0}$ would not be affected by nonpharmacological intervention. Thus, the result would better reflect the true nature of transmissibility within humans for strains with different mutations. Our findings demonstrated that the G614 mutation accelerated the transmission of the COVID-19 virus and also had higher spatial transmissibility, indicating that strains with G614, which were the dominant strains around the world, could spread on a larger scale and be more difficult to control. These results also echo those experimental results in vitro, in which both clinical samples and pseudoviruses with G614 have higher levels of viral RNA and titers compared to those with D614 (5).

As the COVID-19 pandemic spreads and continues, real-time monitoring and evaluation of the impacts of COVID-19 virus strain mutations need to be consistently maintained to provide earlier warnings for the public and provide evidence that supports government-led countermeasures and strategies.

Acknowledgments: We gratefully acknowledge the authors from the originating laboratories and the submitting laboratories where genetic sequence data were generated and shared via GISAID, enabling this research; Strategic Priority Research Program of the Chinese Academy of Sciences (Grant No. XDB29010202, XDB29010102 and XDA19090118); National Science and Technology Major Project (Grant No. 2018ZX10733403, and 2018ZX10713001-010); National Natural Science Foundation of China (NSFC) (Grant No. 32041010 and 31900155); NSFC Outstanding Young Scholars (Grant No. 31822055); and Youth Innovation Promotion Association of CAS (Grant No. 2017122).

doi: $10.46234 / \mathrm{ccdcw} 2020.247$

\# Corresponding authors: Yuhai Bi, beeyh@im.ac.cn; George F. Gao, gaof@im.ac.cn.

${ }^{1}$ CAS Key Laboratory of Pathogenic Microbiology and Immunology, 

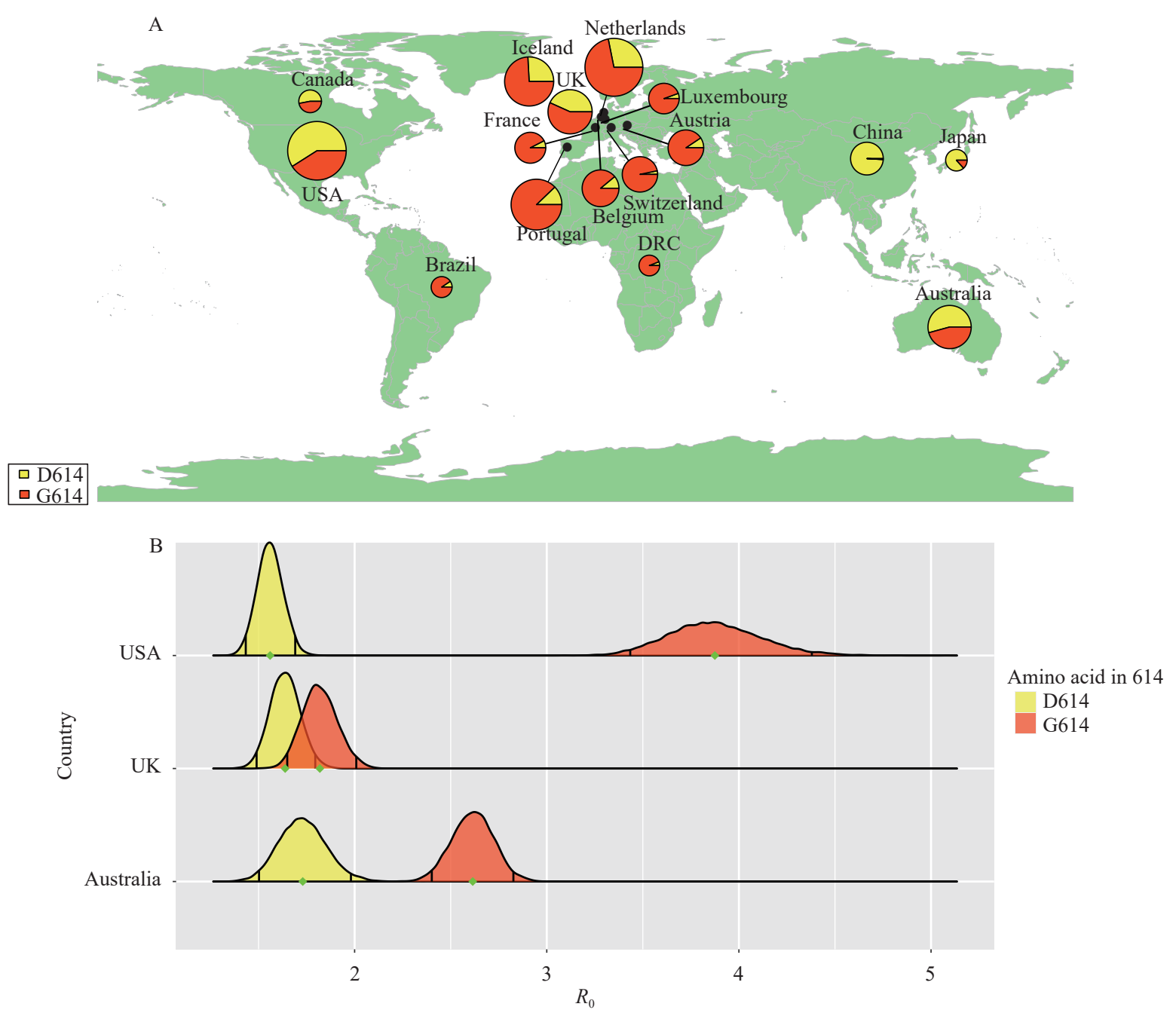

FIGURE 1. The statistics and transmissibility of the COVID-19 virus with D614 and G614 during the early phases of the pandemic within each country. (A) The number of publicly available genomes with D614 and G614 during the first two months after the first confirmed case in each country. The size of the pie chart is associated with the number of genomes. (B) The distribution of $R_{0}$ for D614 and G614 during the first two months after the first confirmed case in each country. The mean of $R_{0}$ is represented by a green point. The black line within the distribution represents the $95 \%$ confidence interval $(\mathrm{Cl})$.

Institute of Microbiology, Center for Influenza Research and Earlywarning (CASCIRE), CAS-TWAS Center of Excellence for Emerging Infectious Diseases (CEEID), Chinese Academy of Sciences, Beijing, China; ${ }^{2}$ University of Chinese Academy of Sciences, Beijing, China.

Submitted: November 21, 2020; Accepted: November 25, 2020

\section{REFERENCES}

1. Jiang SB, Shi ZL, Shu YL, Song JD, Gao GF, Tan WJ, et al. A distinct name is needed for the new coronavirus. Lancet 2020;395(10228):949. http://dx.doi.org/10.1016/S0140-6736(20)30419-0.

2. Tan WJ, Zhao X, Ma XJ, Wang WL, Niu PH, Xu WB, et al. A novel coronavirus genome identified in a cluster of pneumonia cases Wuhan, China 2019-2020. China CDC Weekly 2020;2(4):61 - 2. http://dx.doi.org/10.46234/ccdcw2020.017.
3. Zhu N, Zhang DY, Wang WL, Li XW, Yang B, Song JD, et al. A novel coronavirus from patients with pneumonia in China, 2019. N Engl J Med 2020;382(8):727 - 33. http://dx.doi.org/10.1056/NEJMoa2001 017.

4. Fauver JR, Petrone ME, Hodcroft EB, Shioda K, Ehrlich HY, Watts AG, et al. Coast-to-coast spread of SARS-CoV-2 during the early epidemic in the United States. Cell 2020;181(5):990 - 6.e5. http://dx.doi.org/ 10.1016/j.cell.2020.04.021.

5. Korber B, Fischer WM, Gnanakaran S, Yoon H, Theiler J, Abfalterer W, et al. Tracking changes in SARS-CoV-2 spike: evidence that D614G increases infectivity of the COVID-19 virus. Cell 2020;182(4):812 27.e19. http://dx.doi.org/10.1016/j.cell.2020.06.043.

6. Grubaugh ND, Hanage WP, Rasmussen AL. Making sense of mutation: what D614G means for the COVID-19 pandemic remains unclear. Cell 2020;182(4):794 - 5. http://dx.doi.org/10.1016/j.cell.2020.06.040.

7. Shu YL, McCauley J. GISAID: Global initiative on sharing all influenza data - from vision to reality. EuroSurveillance 2017;22(13):pii=30494 http://dx.doi.org/10.2807/1560-7917.ES.2017.22.13.30494.. 


\section{Supplementary Material}

\section{Data collection, classification, and preliminary process}

In order to minimize the impact of stringent prevention and control measures on the transmission of the COVID-19 virus and strike a balance between the small amount of variation among viral genomes during the early stages of the outbreak and sufficient variation to support this study, we defined the scope of the study to focus on the first 2 months after the start of COVID-19 outbreak within each country. All genomic sequences, their spike protein sequences, and collection dates of the COVID-19 virus were retrieved from GISAID on April 9, 2020. Since there were some countries [e.g. Austria, Brazil, the Democratic Republic of the Congo (DRC), Iceland, Luxembourg, Portugal, and Switzerland] in which the outbreak had not existed for two months before April 9, 2020, we collected additional data for these countries on June 1, 2020 (Table S1). Only complete genomic sequences with high coverage and exact collection dates (accurate to days) were used in this study.Only countries with more than 80 COVID-19 virus genomes were shown in Figure 1A. Genomic and corresponding spike protein sequences from each country were aligned using Mafft v7.310 (1). The genomic sequences were split into two datasets (D and $G$ ) based on the amino acid at 614 of the spike protein sequence (based on Wuhan reference sequence) for each country. To eliminate the potential impact on results due to different regions, we only compared the 2 datasets (G614 and D614) within each country. In addition, only countries where the difference in the number of genomes between the 2 datasets was less than $50 \%$ and both datasets having contained more than 100 genomes were used in the subsequent analysis to minimize the potential impact of the difference in the amount of data on results. In this case, datasets from Australia, the UK, and USA were used in the final analysis. We trimmed uncertain regions in 3' and 5' terminals and also masked 30 sites (Table S2) that are highly homoplastic and have no phylogenetic signal as previous noted (https://virological.org/t/issues-with-sars-cov-2-sequencing-data/473). Finally, we obtained 197, 166, 164, 215, 393, and 273 genomic sequences, with aligned genomic length of 29,381, 29,381, 29,582, 29,582, 29,498, and 29,498 from Australia (D614), Australia (G614), the UK (D614), the UK (G614), USA (D614), and USA (G614), respectively. We used jModelTest v2.1.6 (2) to find the best substitution model for each dataset according to the Bayesian Information Criterion (Table S3). The list of genomic sequences used in this study were openly shared via the GISAID initiative (3) (see the Acknowledgement Table for details).

SUPPLEMENTARY TABLE S1. The time point for each country used in this study.

\begin{tabular}{cccc}
\hline Country & Continent & Date of first confirmed case & The first two months \\
\hline Australia & Oceania & $2020-01-25$ & $2020-03-25$ \\
Austria & Europe & $2020-02-25$ & $2020-04-25$ \\
Belgium & Europe & $2020-02-04$ & $2020-04-04$ \\
Brazil & South America & $2020-02-25$ & $2020-04-25$ \\
Canada & North America & $2020-01-27$ & $2020-03-27$ \\
China & Asia & $2019-12-24$ & $2020-02-24$ \\
DRC & Africa & $2020-03-10$ & $2020-05-10$ \\
France & Europe & $2020-01-24$ & $2020-03-28$ \\
Iceland & Europe & $2020-02-28$ & $2020-04-28$ \\
Japan & Asia & $2020-01-14$ & $2020-03-14$ \\
Luxembourg & Europe & $2020-02-29$ & $2020-04-29$ \\
Portugal & Europe & $2020-03-02$ & $2020-05-02$ \\
Switzerland & Europe & $2020-02-25$ & $2020-04-25$ \\
UK & Europe & $2020-01-31$ & $2020-03-31$ \\
USA & North America & $2020-01-20$ & $2020-03-20$ \\
\hline
\end{tabular}

\section{Reconstruction of dated phylogenies}

Since recombination could impact the evolutionary signal, we first detected the recombination events in these COVID-19 virus genomes by RDP4 (4). No evidence of recombination was found in any dataset. We then used the 

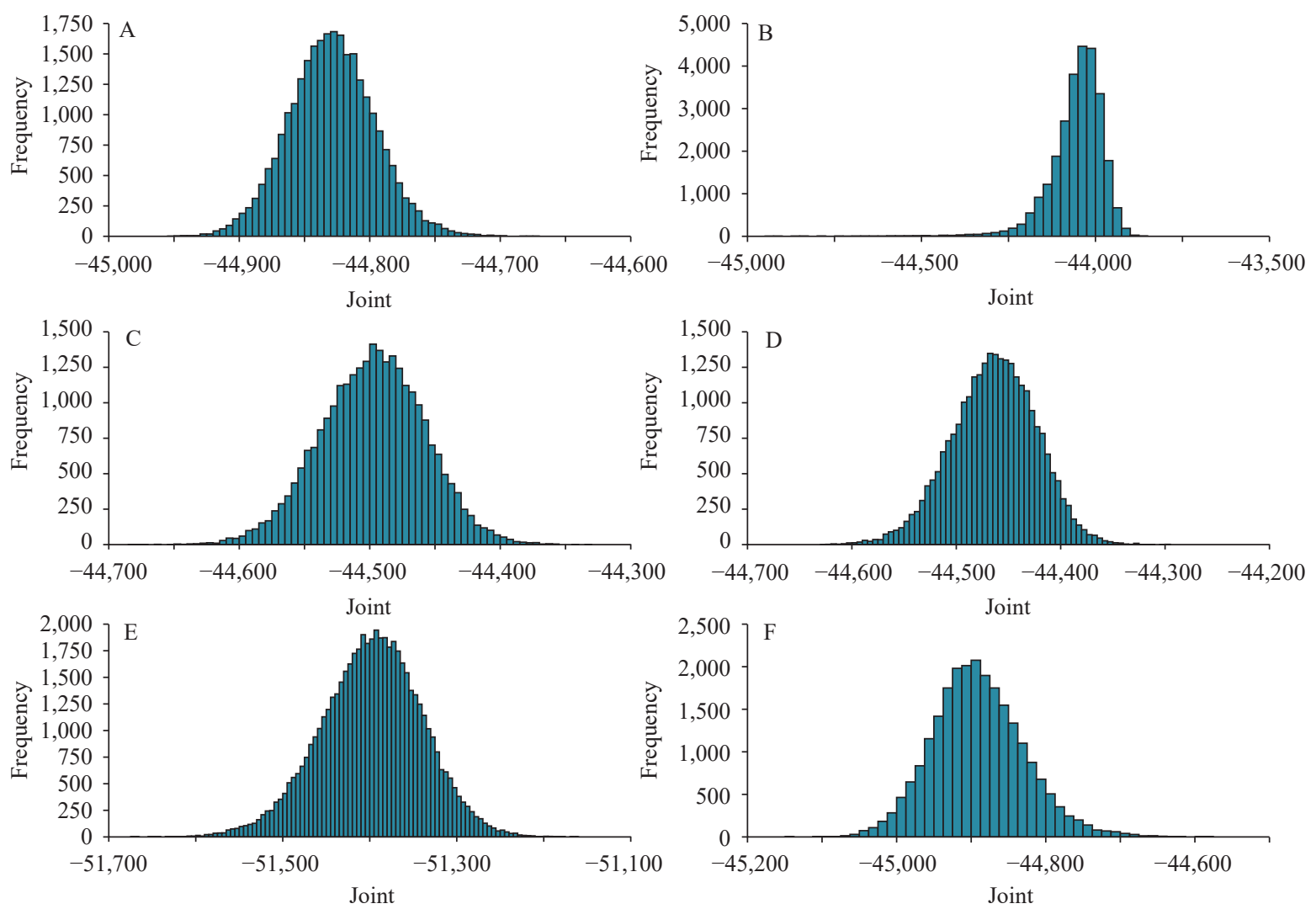

SUPPLEMENTARY FIGURE S1. The posterior distributions of phylogenies in the posterior tree space for each dataset. (A) D614 dataset in Australia; (B) G614 dataset in Australia; (C) D614 dataset in UK; (D) G614 dataset in UK; (E) D614 dataset in USA; (F) G614 dataset in USA

Bayesian Markov Chain Monte Carlo (MCMC) approach implemented in Bayesian Evolutionary Analysis Sampling Trees (BEAST) v1.10.4 (5) to derive an accurate, dated phylogeny for COVID-19 under the best substitution model for each dataset. The result of model comparison was listed in Table S4. Analyses were performed with at least 3 independent replicates of 100 million MCMC steps each and sampling parameters and trees every 10,000 steps. The estimation of the most appropriate combination of molecular clock and coalescent models for Bayesian phylogenetic analysis was determined using both path-sampling (PS) and stepping-stone (SS) models (G). Tracer 1.7.1 (7) was then used to check the convergence of MCMC chains (effective sample size >200) and to compute marginal posterior distributions of parameters after discarding $10 \%$ of the MCMC chain as burn-in (Figure S1). TreeAnnotator was used to summarize a maximum clade credibility (MCC) tree from the posterior distribution of trees after discarding $10 \%$ of the MCMC chain as burn-in (Figure S2). We determined whether there was a sufficient temporal signal in each dataset as it was the prerequisite for getting a reliable inference when performing phylodynamic analysis. Bayesian evaluation of temporal signal (BETS) (8) was used to evaluate the temporal signal in each dataset. BETS relied on the comparison of marginal likelihoods of two models: the heterochronous (with tip date) and isochronous (without tip date) models. Analyses were performed with at least 3 independent replicates of 100 million MCMC steps each and sampling parameters and trees every 10,000 steps with the best substitution model and most appropriate combination of molecular clock and coalescent models determined above for each dataset. The marginal likelihoods were estimated by PS. The Bayes factor (BF) was then calculated based on the likelihoods of two models (heterochronous and isochronous). If the $\log \mathrm{BF}>5$ (heterochronous model against isochronous model), it indicated there were sufficient temporal signals in this dataset. The results of BETS for each country are listed in Table S5. All datasets had log BF>5, suggesting that the temporal signal was sufficiently strong.

\section{Inferring the transmission chain and its parameters}

Because viral genomes were incompletely sampled and the epidemic is still ongoing, TransPhylo v1.3.19 (9) was 
SUPPLEMENTARY TABLE S2. List of 30 masked sites in the COVID-19 virus genome.

\begin{tabular}{|c|c|}
\hline ID & Site \\
\hline 1 & 187 \\
\hline 2 & 1,059 \\
\hline 3 & 2,094 \\
\hline 4 & 3,037 \\
\hline 5 & 3,130 \\
\hline 6 & 4,050 \\
\hline 7 & 6,990 \\
\hline 8 & 8,022 \\
\hline 9 & 10,323 \\
\hline 10 & 10,741 \\
\hline 11 & 11,074 \\
\hline 12 & 11,083 \\
\hline 13 & 13,402 \\
\hline 14 & 13,408 \\
\hline 15 & 14,786 \\
\hline 16 & 15,324 \\
\hline 17 & 19,684 \\
\hline 18 & 20,148 \\
\hline 19 & 21,137 \\
\hline 20 & 21,575 \\
\hline 21 & 24,034 \\
\hline 22 & 24,378 \\
\hline 23 & 25,563 \\
\hline 24 & 26,144 \\
\hline 25 & 26,461 \\
\hline 26 & 26,681 \\
\hline 27 & 28,077 \\
\hline 28 & 28,826 \\
\hline 29 & 28,854 \\
\hline 30 & 29,700 \\
\hline
\end{tabular}

SUPPLEMENTARY TABLE S3. The best substitution model from each dataset.

\begin{tabular}{ccc}
\hline Country & Category & Best substitution model \\
\hline \multirow{2}{*}{ Australia } & D614 & GTR+1 \\
& G614 & GTR \\
UK & D614 & GTR \\
& G614 & GTR \\
USA & D614 & GTR \\
& G614 & GTR \\
\hline
\end{tabular}

used to infer the transmission tree using the MCC dated phylogeny generated above as input, as a previous study demonstrated that using an MCC tree will greatly reduce the impact of phylogenetic uncertainty on the results (10). The generation time (i.e. the time interval from infection onward to transmission, denoted G) of COVID-19 was previously estimated as $7.5 \pm 3.4$ days (11), we used these values to compute the shape and scale parameter of a gamma distribution of $\mathrm{G}$ with the $\mathrm{R}$ package epitrix (12). The distribution of sampling time (i.e. the time gap from 
SUPPLEMENTARY TABLE S4. Log-marginal likelihood estimates from model selection by using the path-sampling (PS) and stepping-stone (SS) approaches.

\begin{tabular}{|c|c|c|c|c|c|}
\hline \multirow{2}{*}{ Country } & \multirow{2}{*}{ Category } & \multirow{2}{*}{ Clock } & \multirow{2}{*}{ Coalescent } & \multicolumn{2}{|c|}{ Log marginal likelihood } \\
\hline & & & & Path-sampling (PS) & Stepping-stone (SS) \\
\hline \multirow{12}{*}{ Australia } & \multirow{6}{*}{ D614 } & Strict & Constant & $-43,161.09724$ & $-43,161.78956$ \\
\hline & & Strict & Exponential & $-43,142.87942$ & $-43,137.39070$ \\
\hline & & Strict & Skyline & $-43,129.81686$ & $-43,131.14703$ \\
\hline & & UCLN & Constant & $-43,161.86138$ & $-43,161.88169$ \\
\hline & & UCLN & Exponential & $-43,159.59768$ & $-43,160.25060$ \\
\hline & & UCLN & Skyline & $-43,137.20113$ & $-43,137.40271$ \\
\hline & \multirow{6}{*}{ G614 } & Strict & Constant & $-42,114.88661$ & $-42,115.21617$ \\
\hline & & Strict & Exponential & $-42,083.25716$ & $-42,082.87311$ \\
\hline & & Strict & Skyline & $-42,077.37285$ & $-42,077.67556$ \\
\hline & & UCLN & Constant & $-42,117.49317$ & $-42,117.88757$ \\
\hline & & UCLN & Exponential & $-42,091.95378$ & $-42,092.27226$ \\
\hline & & UCLN & Skyline & $-42,083.47337$ & $-42,083.78117$ \\
\hline \multirow{11}{*}{ UK } & \multirow{6}{*}{ D614 } & Strict & Constant & $-42,608.42147$ & $-42,608.81099$ \\
\hline & & Strict & Exponential & $-42,597.33016$ & $-42,597.92089$ \\
\hline & & Strict & Skyline & $-42,590.39452$ & $-42,590.98556$ \\
\hline & & UCLN & Constant & $-42,611.70853$ & $-42,611.82690$ \\
\hline & & UCLN & Exponential & $-42,602.15788$ & $-42,602.19886$ \\
\hline & & UCLN & Skyline & $-42,593.34587$ & $-42,593.38936$ \\
\hline & \multirow{6}{*}{ G614 } & Strict & Constant & $-42,723.66227$ & $-42,723.33748$ \\
\hline & & Strict & Exponential & $-42,713.28573$ & $-42,713.22968$ \\
\hline & & Strict & Skyline & $-42,689.38860$ & $-42,689.92583$ \\
\hline & & UCLN & Constant & $-42,728.86399$ & $-42,730.02142$ \\
\hline & & UCLN & Exponential & $-42,717.35538$ & $-42,717.62522$ \\
\hline \multirow{13}{*}{ USA } & & UCLN & Skyline & $-42,697.11254$ & $-42,698.05113$ \\
\hline & \multirow{6}{*}{ D614 } & Strict & Constant & $-44,757.71082$ & $-44,761.16177$ \\
\hline & & Strict & Exponential & $-44,742.61060$ & $-44,746.51211$ \\
\hline & & Strict & Skyline & $-44,716.67090$ & $-44,719.31089$ \\
\hline & & UCLN & Constant & $-44,747.41638$ & $-44,748.70688$ \\
\hline & & UCLN & Exponential & $-44,733.82629$ & $-44,736.89652$ \\
\hline & & UCLN & Skyline & $-44,708.56055$ & $-44,709.34196$ \\
\hline & \multirow{6}{*}{ G614 } & Strict & Constant & $-43,222.54858$ & $-43,222.84280$ \\
\hline & & Strict & Exponential & $-43,196.32220$ & $-43,196.83572$ \\
\hline & & Strict & Skyline & $-43,168.56326$ & $-43,170.73422$ \\
\hline & & UCLN & Constant & $-43,216.36134$ & $-43,217.73716$ \\
\hline & & UCLN & Exponential & $-43,198.82606$ & $-43,199.01257$ \\
\hline & & UCLN & Skyline & $-43,180.20141$ & $-43,178.26833$ \\
\hline
\end{tabular}

infection to detection and sampling) was set to equal the distribution of generation time. We performed TransPhylo with at least 500,000 iterations (and sampling parameters every at least 50 steps) by simultaneously estimating the transmission tree, the proportion of sampling, the within-host coalescent time Neg, and the two parameters of the negative binomial of offspring distribution (which represented the number of secondary cases caused by each infection). All results were generated by discarding the first part of the MCMC chains as burn-in. The MCMC mixing and convergence was assessed based on the effective sample size of each parameter $(>200)$. The estimated parameters for each country and each time point were listed in Table S6. 


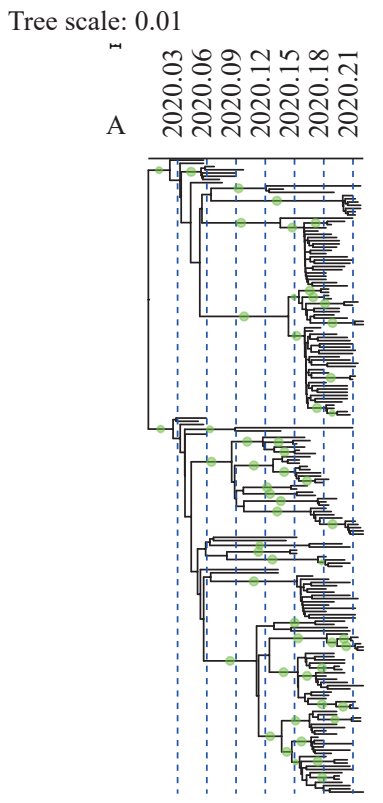

Tree scale: 0.01
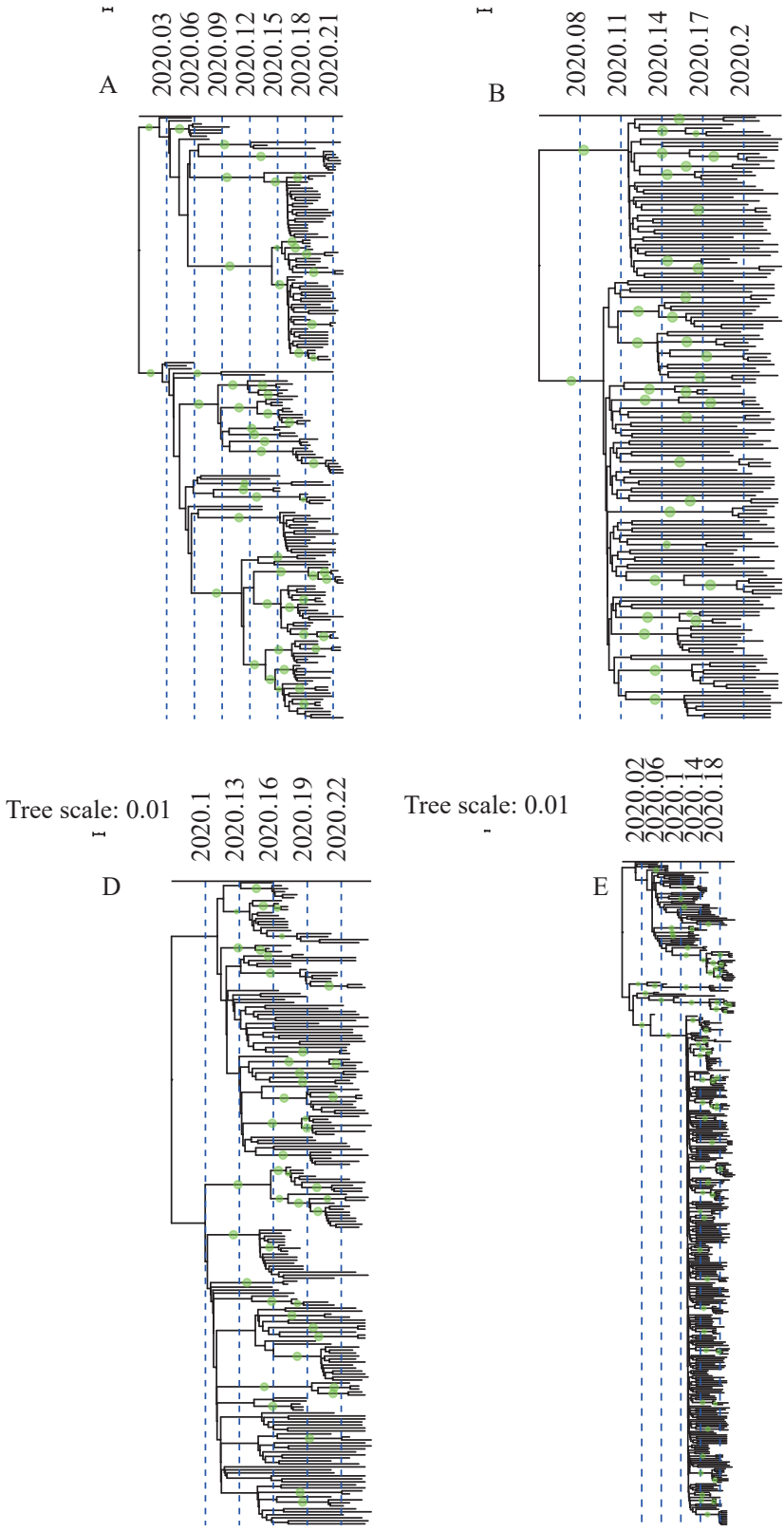
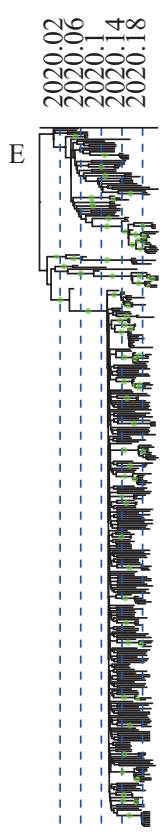

Tree scale: 0.01

t)

๙ิ ๙ิ

C

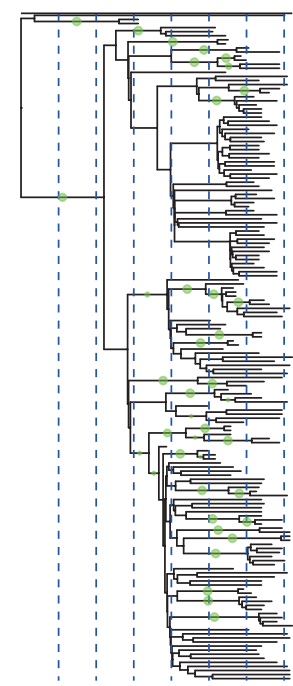

Tree scale: 0.01

드크

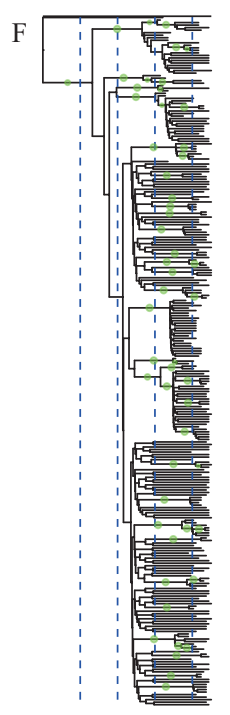

SUPPLEMENTARY FIGURE S2. The maximum clade credibility (MCC) tree of COVID-19 virus during the first two months of the COVID-19 outbreak in each country. Posterior probabilities greater than 0.6 are shown with a green circle. The size of the circle is proportional to the posterior probability. (A) MCC tree for D614 dataset in Australia; (B) MCC tree for G614 dataset in Australia; (C) MCC tree for D614 dataset in UK; (D) MCC tree for G614 dataset in UK; (E) MCC tree for D614 dataset in USA; (F) MCC tree for G614 dataset in USA

SUPPLEMENTARY TABLE S5. Bayesian evaluation for the temporal signal of each dataset.

\begin{tabular}{ccccc}
\hline Country & Category & log likelihood with sampling time (PS) & log likelihood without sampling time (PS) & logBF \\
\hline \multirow{2}{*}{ Australia } & D614 & $-43,129.81686$ & $-43,225.16828$ & 95.35142 \\
& G614 & $-42,077.37285$ & $-42,085.17860$ & 7.80576 \\
\multirow{2}{*}{ UK } & D614 & $-42,590.39452$ & $-42,615.28006$ & 24.88554 \\
& G614 & $-42,689.38860$ & $-42,731.79239$ & 42.40379 \\
\multirow{2}{*}{ USA } & D614 & $-44,708.56055$ & $-44,864.82497$ & 156.26440 \\
& G614 & $-43,168.56326$ & $-43,196.49259$ & 27.92932 \\
\hline
\end{tabular}




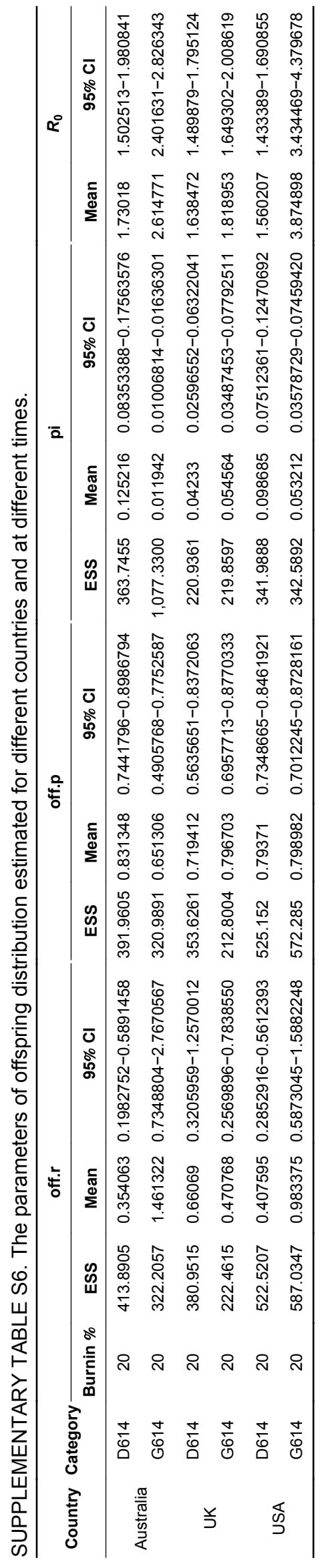




\section{REFERENCES}

1. Katoh K, Misawa K, Kuma K, Miyata T. MAFFT: a novel method for rapid multiple sequence alignment based on fast Fourier transform. Nucleic Acids Res 2002;30(14):3059 - 66. https://www.ncbi.nlm.nih.gov/pmc/articles/PMC4594756/.

2. Darriba D, Taboada GL, Doallo R, Posada D. jModelTest 2: more models, new heuristics and parallel computing. Nature Methods 2012;9(8):772.

3. Elbe S, Buckland-Merrett G. Data, disease and diplomacy: GISAID's innovative contribution to global health. Global Challenges, 1:33-46. https://doi.org/10.1002/gch2.1018.

4. Martin DP, Murrell B, Golden M, Khoosal A, Muhire B. RDP4: detection and analysis of recombination patterns in virus genomes. Virus Evol 2015;1(1):vev003. https:/www.ncbi.nlm.nih.gov/pmc/articles/PMC5014473/.

5. Suchard MA, Lemey P, Baele G, Ayres DL, Drummond AJ, Rambaut A. Bayesian phylogenetic and phylodynamic data integration using BEAST 1.10. Virus Evol 2018;4(1):vey016. https://www.ncbi.nlm.nih.gov/pmc/articles/PMC6007674/.

6. Baele G, Li WLS, Drummond AJ, Suchard MA, Lemey P. Accurate model selection of relaxed molecular clocks in Bayesian phylogenetics. Mol Biol Evol 2013;30(2):239 - 43. https://www.ncbi.nlm.nih.gov/pmc/articles/PMC3548314/.

7. Rambaut A, Drummond AJ, Xie D, Baele G, Suchard MA. Posterior summarization in Bayesian phylogenetics using Tracer 1.7. Syst Biol 2018;67(5):901 - 4. http://dx.doi.org/10.1093/sysbio/syy032.

8. Duchene S, Lemey P, Stadler T, et al. Bayesian evaluation of temporal signal in measurably evolving populations. Mol Biol Evol 2020. https://www. ncbi.nlm.nih.gov/pmc/articles/PMC7454806/.

9. Didelot X, Fraser C, Gardy J, Colijn C. Genomic infectious disease epidemiology in partially sampled and ongoing outbreaks. Mol Biol Evol 2017;34(4):997 - 1007. https://www.ncbi.nlm.nih.gov/pmc/articles/PMC5850352/.

10. Wang L, Didelot X, Yang J, Wong G, Shi Y, Liu WJ, et al. Inference of person-to-person transmission of COVID-19 reveals hidden super-spreading events during the early outbreak phase. Nat Commun 2020;11(1):5006. http://dx.doi.org/10.1038/s41467-020-18836-4.

11. Li Q, Guan X, Wu P, Wang XY, Zhou L, Tong YQ, et al. Early transmission dynamics in Wuhan, China, of novel coronavirus-infected pneumonia. N Engl J Med 2020;382(13):1199 - 207. http://dx.doi.org/10.1056/NEJMoa2001316.

12. Thibaut J, Anne C. Epitrix: small helpers and tricks for epidemics analysis. 2019. https://CRAN.R-project.org/package=epitrix. 